\title{
Decaying 2D Turbulence in Bounded Domains: Influence of the Geometry
}

\author{
Kai Schneider ${ }^{1}$ and Marie Farge ${ }^{2}$ \\ 1 MSNM-CNRS \& CMI, Université de Provence, Marseille, France \\ kschneid@cmi.univ-mrs.fr \\ 2 LMD-CNRS, Ecole Normale Supérieure, Paris, France \\ farge@lmd.ens.fr
}

\begin{abstract}
We present direct numerical simulation of two-dimensional decaying turbulence in wall bounded domains. The Navier-Stokes equations are solved in a periodic square domain using the vorticity-velocity formulation. The bounded domain is imbedded in the periodic domain and the no-slip boundary conditions on the wall are imposed using a volume penalisation technique. The numerical integration is done with a Fourier pseudo-spectral method combined to a semi-implicit time discretization with adaptive time stepping. We study the influence of the geometry of the domain on the flow dynamics and in particular on the long time behaviour of the flow. We consider different geometries, a circle, a square, a triangle and a torus and we show that the geometry plays a crucial role for the decay scenario.
\end{abstract}

Key words: 2D turbulence, penalisation, bounded geometry, long time decay

\section{Introduction}

Two-dimensional turbulence in wall bounded domains has many applications in geophysical flows, e.g. in oceanography and in planetary flows. Direct numerical simulations of two-dimensional turbulence in circular and square domains can be found, e.g. in $[1,2,3]$. The aim of the present paper is to study the influence of the geometry of the domain on the flow dynamics and in particular on the long time behaviour of the flow. Typically one observes the formation of stable large scale structures which persist for a long time before they are finally dissipated. Viscous dissipation is the dominant mechanism of these final states, as the nonlinear term in the Navier-Stokes equations is depleted when there is a functional relationship between the streamfunction and the vorticity. Late states of decaying two-dimensional flows in periodic boxes have for instance been investigted in [4]. Here we study the final states of wall bounded flows considering different geometries, a circle, a square, a triangle and a torus. Several theoretical predictions of the long time behaviour of twodimensional flows can be found, e.g. in the book of Davidson [5]. Variational principles for predicting the final state are based on conservation of energy 
$E$ and the decay of enstrophy $Z$. In this heuristic approach $Z$ is minimized under constraint of conservation of $E$ [6]. Another variational hypothesis is motivated by statistical mechanics. A measure of mixing can be introduced which leads to the definition of an entropy. The final states correspond to a maximum of entropy as turbulence maximizes mixing [7, 8, 9]. Another approach based on viscous eigenmodes of the Stokes flow has been used in [10] to predit the self-organisation of two-dimensional flows in a slip-free box, and for the no-slip case in [11].

\section{Numerical Scheme}

The Navier-Stokes equations are solved in a double periodic square domain of size $L=2 \pi$ using the vorticity-velocity formulation. The bounded domain is thus imbedded in the periodic domain and the no-slip boundary conditions on the wall $\partial \Omega$ are imposed using a volume penalisation method. The physical idea is to model the solid wall as a porous medium and to compute the flow in a larger domain with two regions of different permeability. A mathematical analysis of the method is given in [12], proving its convergence towards the Navier-Stokes equations with no-slip boundary conditions. The governing equations in vorticity-velocity formulation are,

$$
\partial_{t} \omega+\mathbf{u} \cdot \nabla \omega-\nu \nabla^{2} \omega+\nabla \times\left(\frac{1}{\eta} \chi \mathbf{u}\right)=0
$$

where $\mathbf{u}=(u, v)$ is the divergence-free velocity field, i.e. $\nabla \cdot \mathbf{u}=\partial_{x} u+\partial_{y} v=0$, $\omega=\partial_{x} v-\partial_{y} u$ the vorticity, $\nu$ the kinematic viscosity and $\chi(\mathbf{x})$ a mask function which is 0 inside the fluid, i.e. for $\mathbf{x} \in \Omega$, and 1 inside the solid wall. The penalisation parameter $\eta$ is chosen to be sufficiently small $\left(\eta=10^{-3}\right)$ [13]. The numerical technique we use here is based on a dealiased Fourier pseudospectral method with semi-implicit time discretization and adaptive time-stepping using a CFL condition for the maximum velocity. Details on the code together with its numerical validation can be found in [13].

The energy $E$, enstrophy $Z$ and palinstrophy $P$ of the flow can be defined as $[14]$

$$
E=\frac{1}{2} \int_{\Omega}|\mathbf{u}|^{2} d \mathbf{x}, Z=\frac{1}{2} \int_{\Omega}|\omega|^{2} d \mathbf{x}, P=\frac{1}{2} \int_{\Omega}|\nabla \omega|^{2} d \mathbf{x}
$$

respectively.

The energy dissipation is given by $d_{t} E=-2 \nu Z$ and the enstrophy dissipation by

$$
d_{t} Z=-2 \nu P+\nu \oint_{\partial \Omega} \omega(\mathbf{n} \cdot \nabla \omega) d s,
$$

where $\mathbf{n}$ denotes the outer normal vector with respect to the boundary of the domain $\partial \Omega$. The surface integral in (3) reflects the enstrophy production at the wall involving the vorticity and its gradients which is not present in the periodic case. 


\section{Numerical Results}

Starting with the same random initial conditions, i.e. a correlated Gaussian noise with an energy spectrum $E(k) \propto k^{4}$, we compute the flow evolution in four different geometries for initial Reynolds numbers, $R e=2 D \sqrt{2 E} / \nu$, of about 1000 (where $D$ denotes corresponds to the domain size). Figure 1 shows the vorticity fields at early, intermediate and late times for a circular, a square, a triangle and a torus geometry. All flows organize into large scale structures before more or less quasi-steady states form. For the circlular geometry (Fig. 1, top) we observe the transition via a tripole structure, before the final state, a negative circular vortex surrounded by a vortex ring of positive vorticty, forms. The final state of the toroidal geometry (Fig. 1, bottom) corresponds to two vortex rings, a positive one enclosed by a negative one. The transition phase shows a triangularly shaped vortical structure surrounded by three positive vortices. For the triangle and the square domain we see that the final state is note yet completely reached. However, we find also a negative circular vortex in the center which is encircled by a positive vorticity ring, which seems not yet be completely relaxed.

Figure 2 presents the decay of different integral quantities, energy (left), enstrophy (middle) and palinstrophy (right) for the four geometries. All quantities exhibit at early times a rapid monotonuous decay, except for the square and triangular geometry where we observe an oscillatory behaviour in the palinstrophy decay, however less pronouned for the latter case. These oscillations come from the enstrophy production at the boundary. At later times we find for all geometries and all quantities an exponential decay behaviour with slopes depending on the geometry. The square domain shows the slowest decay of all quantities. The decay is increasing in the following order: torus, triangle and the circle exhibits the fastest decay. The fact that the circular case decays fastest results from the smooth boundary, i.e. no corners are present and hence the enstrophy production at the wall is much less pronounced than for the other cases. 

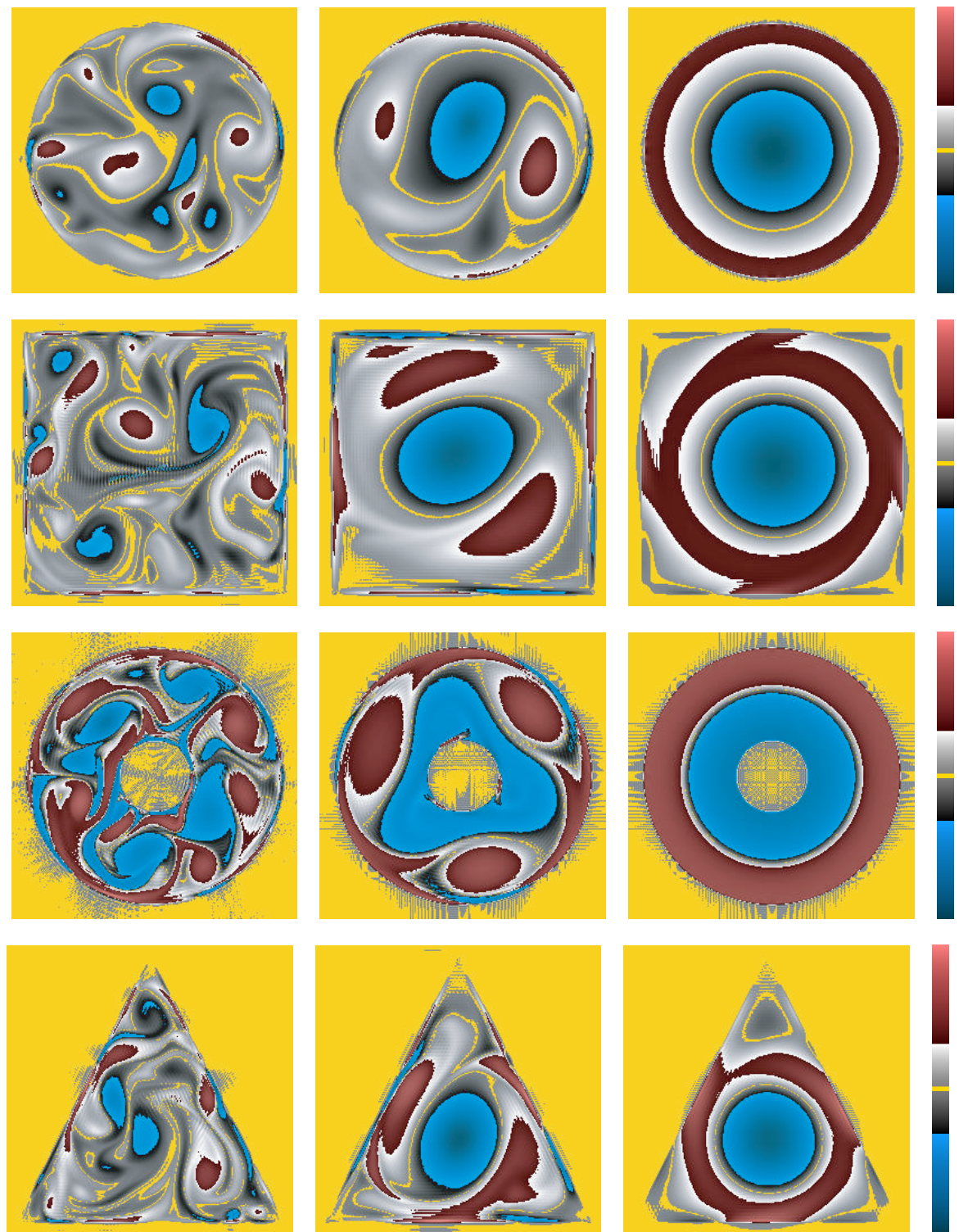

Fig. 1. 2d decaying turbulence in bounded domains. Vorticity fields at early (left), intermediate (middle) and late times (right). From top to bottom: circle, square, triangle and torus. 

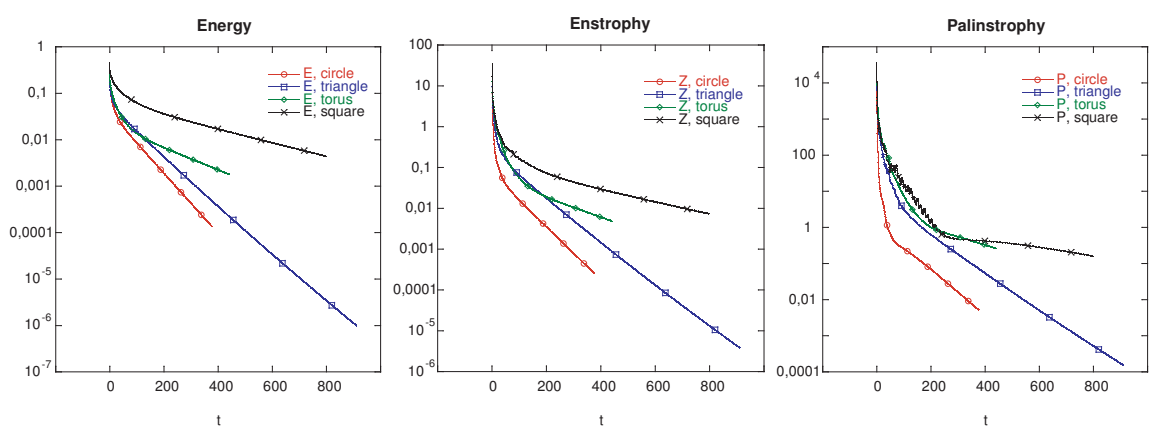

Fig. 2. 2d decaying turbulence in bounded domains. Decay of energy (left), enstrophy (middle) and palinstrophy (right) for the different geometries: circle, square, triangle and torus.

\section{Conclusion}

In conclusion, we have shown, by means of DNS of wall bounded flows in different domains, that no-slip boundaries play a crucial role for decaying turbulent flows. At early times we observe a decay of the flow which leads to self-organisation and the emergence of vortices in the bulk flow, similarly to flows in double periodic boxes. At later times larger scale structures form which depend on the domain geometry, and which finally relax to quasi-steady states. More details on the high Reynolds number simulation for shorter times can be found in [3]. Current work deals with comparison of the final states computed here with predictions of the viscous eigenmodes of the Stokes flow for the different geometries.

Acknowledgements: We thankfully acknowledge financial support from Nagoya University and from the contract CEA/EURATOM $n^{0}$ V.3258.001.

\section{References}

1. Clercx HJH, Nielsen AH, Torres DJ, Coutsias EA (2001) Eur J Mech B - Fluids 20:557-576

2. Li S, Montgomery D, Jones B (1997) Theor Comput Fluid Dyn 9:167-181

3. Schneider K, Farge M (2005) Phys Rev Lett 95:244502

4. Segre E, Kida S (1998) Fluid Dyn Res 23:89-112

5. Davidson P (2004) Turbulence. An Introduction for Scientists and Engineers Oxford University Press

6. Leith CE (1984) Phys Fluids, 27(6):1388-1395

7. Joyce G, Montgomery D (1973) J Plasma Phys 10:107

8. Montgomery D, Joyce G (1974) Phys Fluids 17:1139-1145

9. Robert R, Sommeria J (1991) J Fluid Mech 229:291-310 
10. Kondoh Y, Yoshizawa M, Nakano A, Yabe T (1996) Phys Rev E 54(3):30173020

11. van de Konijnenberg JA, Flor JA, van Heijst GJF (1998) Phys Fluids 10(3):595606

12. Angot P, Bruneau CH, Fabrie P (1999) Numer Math 81:497-520

13. Schneider K (2005) Comput Fluids 34:1223-1238

14. Kraichnan RH, Montgomery D (1980) Rep Progr Phys 43:547-619

15. Montgomery D, Matthaeus WH, Stribling WT, Martinez D, Oughton S (1992) Phys Fluids A 4:3-6 